\title{
Case Report: Early Treatment With Chenodeoxycholic Acid in Cerebrotendinous Xanthomatosis Presenting as Neonatal Cholestasis
}

\begin{abstract}
Irene Degrassi ${ }^{1}$, Chiara Amoruso ${ }^{1}$, Giuseppe Giordano ${ }^{2}$, Marina Del Puppo ${ }^{3}$, Andrea Mignarri ${ }^{4}$, Maria Teresa Dotti ${ }^{4}$, Mauro Naturale ${ }^{2}$ and Gabriella Nebbia ${ }^{1 *}$

${ }^{1}$ Pediatric Intermediate Care Unit, Fondazione IRCCS Ca' Granda Ospedale Maggiore Policlinico, Milan, Italy, ${ }^{2}$ Mass Spectrometry Laboratory, Women's and Children' Health Department, University of Padua, Fondazione Istituto di Ricerca Pediatrica Città della Speranza, Padova, Italy, ${ }^{3}$ School of Medicine and Surgery, University of Milano-Bicocca, Milan, Italy, ${ }^{4}$ Unit of Neurology and Neurometabolic Diseases, Departement of Neurological and Motor Sciences, University of Siena, Siena, Italy
\end{abstract}

Background: Cerebrotendinous xanthomatosis (CTX) is an inborn disorder of bile acid synthesis which causes progressive accumulation of toxic metabolites in various organs, particularly in brain and tendons. Most cases are diagnosed and treated in the second or third decade of life, when neurological involvement appears. We describe a case of CTX presenting as neonatal cholestasis.

OPEN ACCESS

Edited by:

Pietro Vajro,

University of Salerno, Italy

Reviewed by:

Emmanuel Jacquemin

Bicêtre Hospital, France

Hidde Huidekoper

Erasmus Medical Center, Netherlands

${ }^{*}$ Correspondence:

Gabriella Nebbia

gabriella.nebbia@policlinico.mi.it

Specialty section:

This article was submitted to

Pediatric Gastroenterology,

Hepatology and Nutrition,

a section of the journal

Frontiers in Pediatrics

Received: 16 April 2020

Accepted: 05 June 2020

Published: 16 July 2020

Citation:

Degrassi I, Amoruso C, Giordano G

Del Puppo M, Mignarri A, Dotti MT, Naturale M and Nebbia G (2020) Case

Report: Early Treatment With

Chenodeoxycholic Acid in

Cerebrotendinous Xanthomatosis

Presenting as Neonatal Cholestasis.

Front. Pediatr. 8:382.

doi: 10.3389/fped.2020.00382
Results: The child presented cholestasis at 2 months of life. In the following months jaundice slowly disappeared, with a normalization of bilirubin and aminotransferases, respectively, at 6 and 8 months. A LC-Mass Spectrometry of the urines showed the presence of cholestanepentols glucuronide, which led to the suspicion of cerebrotendinous xanthomatosis. The diagnosis was confirmed by the dosage of cholestanol in serum and the molecular genetic analysis of the CYP27A1 gene. Therapy with chenodeoxycholic acid (CDCA) was started at 8 months and is still ongoing. The child was monitored for 13 years by dosage of serum cholestanol and urinary cholestanepentols. A strictly biochemical and neurological follow up was performed and no sign of neurological impairment was observed.

Conclusions: Prompt diagnosis and treatment of CTX presenting as neonatal cholestasis may prevent further neurological impairment.

Keywords: inborn errors of bile acid metabolism, cerebrotendinous xanthomatosis, neonatal cholestasis, chenodeoxycholic acid, CYP27A1 gene, cholestanol

\section{INTRODUCTION}

Cerebrotendinous xanthomatosis (CTX) is an autosomal recessive lipid storage disorder with a defect in bile acid synthesis $(1,2)$. It is caused by mutations in gene CYP27A1 (OMIM*606530), that encodes the mitochondrial sterol 27-hydroxylase enzyme (EC 1.14.13.15), catalyzing the first step in the process of cholesterol side chain oxidation. The synthesis of primary bile acids cholic acid (CA) and chenodeoxycholic acid (CDCA) is impaired: as a result, the normal feedback inhibition of $7 \alpha$-hydroxylase is disrupted with consequent production of toxic metabolites (mainly cholestanol) and bile alcohol glucuronides, detectable, respectively, in plasma and urines (Figure 1). Cholestanol and cholesterol accumulate in particular in nervous system, eyes and tendons (3). 


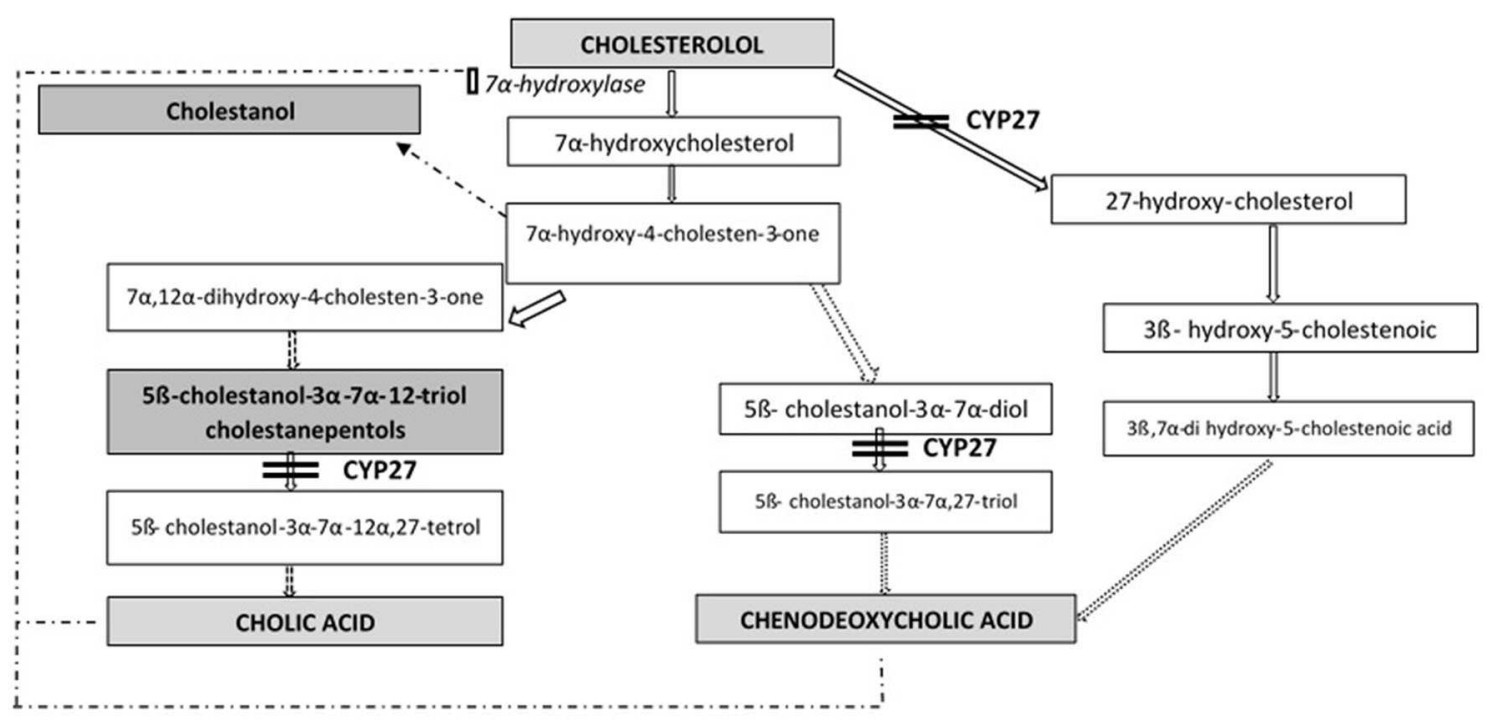

FIGURE 1 | Bile acid synthesis in cerebrotendinous xanthomatosis.

The estimated prevalence of CTX is 1:50.000 (4), with high variability among patients regarding age of onset and type of symptoms, since clinical manifestations appear along with the progressive accumulation of toxic metabolites (5). In infancy CTX may cause cholestatic jaundice, that usually spontaneously resolves $(6,7)$, so that the disease may be overlooked. During childhood cataract, chronic diarrhea and psychomotor retardation can be the first manifestations $(8,9)$; however, as the previous symptoms are aspecific, the diagnosis is often delayed until the appearance in adulthood of tendon xanthomas and the onset of neurological symptoms (spastic paresis, cerebellar ataxia, polyneuropathy, cognitive difficulties, dementia) (10).

Therapy with primary bile acids (chenodeoxycholic acid and cholic acid) is the standard treatment: it replaces the deficiency of primary bile acids and normalizes the levels of cholestanol, bile alcohols and cholesterol, with improvement in clinical, and biochemical manifestations $(11,12)$.

Here, we describe a long term follow-up of a girl with CTX, presenting as neonatal cholestasis at 2 months of life, promptly treated with CDCA since the age of 8 months.

\section{CASE DESCRIPTION}

The patient was a female baby of caucasian origin, born after an uncomplicated pregnancy and delivery. Neonatal weight was $3.100 \mathrm{Kg}$. The parents were not consanguineous and did not present any ophthalmological, neurological, cardiac or hepatic disease. She was breast-fed with normal growth and development

Abbreviations: CA, cholic acid; CTX, cerebrotendinous xanthomatosis; CDCA, chenodeoxycholic acid; CYP27A1, sterol 27-hydroxylase; MS, Mass Spectrometry; SPE, solid phase extraction; FIA, flow injection analysis; UDCA, ursodeoxycholic acid; EEG, Electroencephalogram; MRI, magnetic resonance imaging. until the second month of life, when jaundice was noticed, so that the patient was referred to the Pediatric Department of our hospital. Physical examination showed no dysmorphic features, severe jaundice and a slightly firm liver, palpable at $2 \mathrm{~cm}$ from the costal edge. No history of steathorrea, dark urine or acholic stools was reported. Blood tests showed high levels of total $(13.56 \mathrm{mg} / \mathrm{dl})$ and conjungated bilirubin $(8.79$ $\mathrm{mg} / \mathrm{dl}$ ), elevation of transaminases (AST $473 \mathrm{UI} / \mathrm{l}$; ALT 530 UI/l), with normal gamma-GT, alkaline phosphatase, serum bile acids and INR. Ophtalmoscopic examination and abdominal ultrasound scan were normal. According to current protocols in cholestatic patients (13), we have started early supplementation with vitamin $\mathrm{A}, \mathrm{D}, \mathrm{E}$ and $\mathrm{K}$ since the age of 2 months to avoid complications related to fat soluble vitamins deficiency. Taking into account clinical and biochemical findings, main causes of neonatal cholestasis were excluded.

Considering the clinical picture of unexplained prolonged cholestasis, a percutaneous liver biopsy was performed, showing aspecific features of idiopathic hepatocellular cholestasis such as pronounced lobular disarray, giant-cells, bile pigment within hepatocytes, with slightly enlarged portal tracts in the absence of bile duct proliferation.

Therapy with ursodeoxycholic acid (UDCA) was started at 3 months of age at the dosage of $15 \mathrm{mg} / \mathrm{Kg}$. In the following months jaundice slowly disappeared, with a normalization of bilirubin and transaminases, respectively, at 6 and 8 months. At the age of 7 months in view of the presence of intrahepatic cholestasis with normal colored stools, absence of pruritus, normal serum bile acids and gGT, urinary bile acids profile was performed in the suspicion of bile acid synthesis defects. The analysis showed the presence of $5 ß$-cholestanol-3 $\alpha-7 \alpha-12$-triol (cholestanepentols glucuronide) at the value of $38,4 \mathrm{ratio} / \mathrm{mM}$ creat (normal value: $0,86 \mathrm{ratio} / \mathrm{mM}$ creat), while serum cholestanol had a value of $3,140 \pm 55 \mu \mathrm{g} / \mathrm{dl}$ (normal control value $470 \mu \mathrm{g} / \mathrm{dl}$ ); these findings 
allowed a suspicion of CTX. Mutation analysis of CYP27A1 showed homozygosity for a deletion of $1,9 \mathrm{~Kb}$ with loss of exon $7,8,9$ on chromosome 2 q33-qter, leading to a definite diagnosis.

Therapy with UDCA was stopped and CDCA was started at 8 months of age. The initial dose was $10 \mathrm{mg} / \mathrm{Kg} /$ day, increased until $15 \mathrm{mg} / \mathrm{Kg} /$ day. A parallel, constant decrease of cholestanol in plasma was noticed: from $3,140 \mu \mathrm{g} / \mathrm{dl}$ to $600 \mu \mathrm{g} / \mathrm{dl}$ after 14 months of treatment (Figure 2). The patient has been followed for 13 years: in the absence of clinical manifestations and in the presence of normal laboratory values, the patient was strictly monitored with periodic assessment of plasma cholestanol and urinary cholestanepentols glucuronide to monitor silent accumulation of abnormal metabolites with consequent therapy adjustments (Figure 2). The values remained in the normal limits throughout the years and the therapy was well-tolerated, with no evidence of clinical or biochemical side effects. As regards hepatic involvement, serum liver function tests remained always in the normal limits. Periodic abdominal ultrasound scans were normal and transient elastography, performed at the age of eleven, showed normal liver stiffness $(4.1 \mathrm{Kpa})$. Concerning possible neurologic involvement, annual electroencephalograms (EEG), neurological examinations and ophthalmoscopy controls were performed without detection of complications, as well as a brain magnetic resonance (MRI) performed at the age of 8 years. No xanthomas were detected in the Achilles tendons or other regions. Finally, the child showed a normal growth and cognitive development, with excellent school results and physical performance.

\section{MATERIALS AND METHODS}

\section{Urine Bile Acids}

The qualitative and quantitative analysis was made by liquid chromatography-tandem mass spectrometry (LC-MS/MS). The urine sample used was calculated in base at $30 \mathrm{mg}$ of creatinine (Cr) was considered consistent with effective suppression of the abnormal pathway. Urine samples were collected from the patients and stored at $-20^{\circ} \mathrm{C}$ until analysis was carried out. The concentration of bile acids in the urine was corrected according to the $\mathrm{Cr}$ concentration and expressed as micromoles

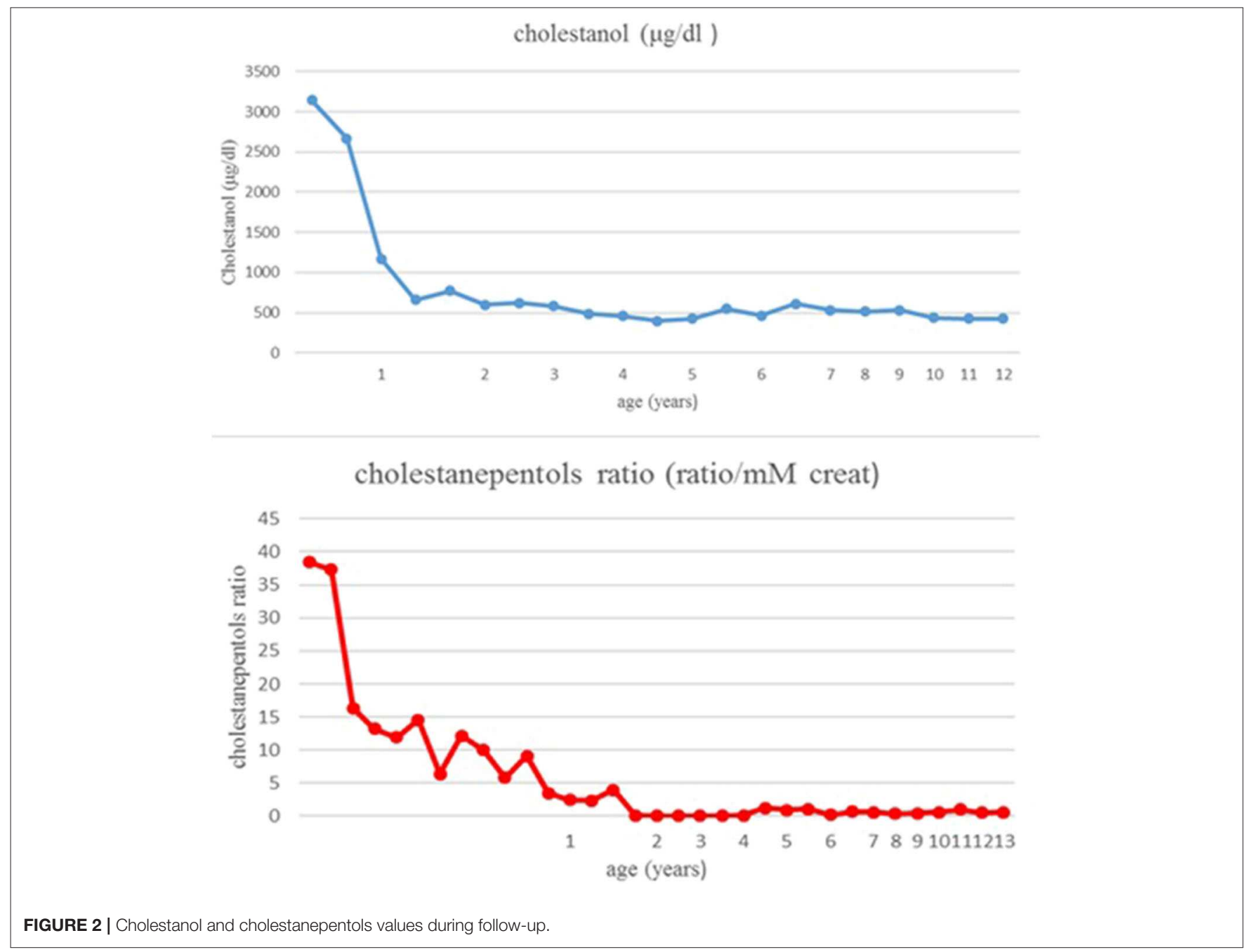


per millimole of Cr. For extraction of bile acids from urine we used "OASIS HLB" Waters solid-phase extraction cartridges $(1 \mathrm{~mL})$. The cartridges were prewashed with methanol and distilled water. The urine sample, previously normalized for the Cr concentration, was passed through a cartridge with $50 \mu \mathrm{L}$ of internal standard (100 mM of CA and CDCA conjugated or not with glycine and taurine, labeled with four atoms of deuterium). The bile acids and alcohols were eluted with 400 $\mu \mathrm{L}$ of methanol, taken to dryness by a stream of $\mathrm{N} 2$, and then diluted with $100 \mu \mathrm{L}$ of a solution of $50 \%$ acetonitrile. The sample was analyzed by the mass spectrometer micromass QUATTRO Ultima interfaced with HPLC HT Waters 2790 by electrospray ionization. The analysis was performed by direct injection (without a column). The negative ion mass spectra of the elutes were recorded in the mass range of 300 to $700 \mathrm{Da}$, which represents the mass ranges of the different urinary bile acids. Data were elaborated by neolynx software (MassLynx, Waters Corp, Milford, MA).

\section{Plasma Cholestanol}

Serum levels of cholestanol were assayed as previously described (14). Briefly, deuterated cholestanol $(1 \mu \mathrm{g})$ as internal standard was added to $0.2-\mathrm{mL}$ serum samples and alkaline hydrolysis was carried out with $1 \mathrm{~mL} 1 \mathrm{~N} \mathrm{NaOH}$ in $90 \%$ ethanol at $60^{\circ} \mathrm{C}$ for $90 \mathrm{~min}$ under nitrogen. After addition of saline $(1 \mathrm{~mL})$, sterols were extracted with $2 \mathrm{~mL}$ petroleum ether and taken to dryness under a stream of nitrogen. The extracted sterols were then converted into their pentafluorobenzoyl derivatives (PFB), as described by (15), and purified by HPLC separation in order to remove excess cholesterol from lipid extract. The PFB-cholestanol fraction of each sample was collected and, after solvent evaporation, it was dissolved in $25 \mu \mathrm{l}$ of toluene for GC-MS analysis. Analysis of sterols was carried out using a Thermo Finnigan GC-Q instrument (Waltham, MA, USA). For sterols quantification we focused specific ions: $\mathrm{m} / \mathrm{z} 215$ and 427 for PFB-cholestanol, m/z 219 and 431 for deuterated PFB-cholestanol. Calibration curves were prepared by spiking plasma with a fixed amount of internal standard and increasing amounts of cholestanol. These samples were treated and analyzed as the experimental samples. Concentrations were calculated on the basis of the slope of the standard curve and on the peak area ratio (sterol/IS) found in the sample. The assay results were linear $(r>0.98)$ in the tested ranges.

\section{DISCUSSION}

We describe a case of cerebrotendinous xanthomatosis presenting as neonatal cholestasis with normal levels of gammaGT in the first year of life. Other cholestatic infants with spontaneous recovery have been described $(6,16-18)$ and this benign clinical picture could explain why specific investigations for CTX are seldom performed, with an underdiagnosis in pediatric age. During the first months of life the alternative pathway of bile acid synthesis is important while the major pathway is immature. Since CYP27A1 is intensively involved in the alternative pathway, this may explain in part neonatal cholestasis (19). When the major pathway becomes mature, then cholestasis may resolve, as in our patient. Fatal neonatal cholestasis have been exceptionally reported $(7,20)$, but the presence of co-infection with CMV might have played an important role in this negative evolution.

The two primary bile acids (CDCA or CA) have been used as a treatment of this condition $(5,21)$, but most authors consider CDCA the treatment of choice, both in adults $(4,5,22)$ and in children $(8,23)$. CA has been successfully used in some patients with CTX and in large cohorts of patients with other bile acid synthesis and zellweger spectrum disorders $(12,16,24)$. Chenodeoxycholic acid seems to have a better biochemical profile as cholic acid levels are not reduced in CTX, while CDCA levels are deficient (25). Besides chenodeoxycholic acid has an effect on feedback inhibition of HMG CoA reductase, thereby suppressing cholesterol production and xanthomas' formation (26).

In an infant treated before the onset of cholestasis, CDCA has been reported as hepatotoxic (18). In this patient cholestasis developed after 6 weeks of treatment and liver enzymes returned to normal values within 3 months after cessation of CDCA. It must be noticed that jaundice with spontaneous resolution can be a manifestation of the disease, as in our patient, so that the relationship with the treatment is uncertain, in this patient hepatitis did not recur on re-challenge with $\operatorname{CDCA}(4,18)$. Both CDCA and CA have been shown safe during pregnancy $(5,11,27,28)$.

In our patient CDCA was started at the age of 8 months; at this time her cholestanol level was already very high and dropped down under therapy. During the whole period of follow-up no further detection of abnormal urinary and plasma metabolites was noticed. In 13 years of follow-up the patient did not show any symptoms, with a completely normal neurological development, as well as persistently normal laboratory values; moreover, no collateral effect was noticed. We can hypothesize that the early beginning of the therapy, with rapid, persistent decrease of the toxic molecules, has prevented clinical complications. It has been previously demonstrated that starting treatment as early as possible can improve and even avoid the onset of neurological involvement $(10,29)$. Taking into account this observation, some authors suggest to include CTX in newborn screening $(30,31)$.

We suggest to look for the presence of cerebrotendinous xanthomatosis in patients with unexplained prolonged neonatal cholestasis with normal levels of gamma-GT and serum bile acids, in order to increase the rate and timing of new diagnoses and to start a prompt treatment. Since accumulation of toxic metabolites is progressive but may be clinically silent, we recommend a strict metabolic monitoring during the therapy with consequent dose adjustments. In conclusion, considering on one side the high frequency of severe clinical manifestations in CTX, and on the other side the availability of an effective and safe therapy, our experience stresses the importance of an early diagnosis and treatment, to prevent irreversible systemic and neurological damage. 


\section{DATA AVAILABILITY STATEMENT}

Publicly available datasets were analyzed in this study. The raw data supporting the conclusions of this article will be made available by the authors, without undue reservation, to any qualified researcher.

\section{ETHICS STATEMENT}

Ethical review and approval was not required for the study on human participants in accordance with the local legislation and institutional requirements. Written informed consent to participate in this study was provided by the participants' legal guardian/next of kin.

\section{TAKE HOME MESSAGE}

Cerebrotendinous xanthomatosis should be suspected in patients with unexplained neonatal cholestasis, even with spontaneous recovery. Considering on one side the high frequency of severe clinical manifestations in CTX, and on the other side the availability of an effective and safe therapy with CDCA, an early

\section{REFERENCES}

1. Van Bogaert L, Scherer HJ, Froehlich A, Epstein E. Une deuxieme observation de cholesterinose tendineuse symetrique avec symptomes cerebraux. Ann Med. (1937) 42:69-101.

2. Clayton PT. Disorders of bile acid synthesis. J Inherit Metab Dis. (2011) 34:593-604. doi: 10.1007/s10545-010-9259-3

3. Heubi JE, Setchell KDR, Bove KE. Inborn errors of bile acid metabolism. Clin Liver Dis. (2018) 22:671-87. doi: 10.1016/j.cld.2018.06.006

4. Verrips A, Dotti MT, Mignarri A, Stelten BML, Verma S, Federico A. The safety and effectiveness of chenodeoxycholic acid treatment in patients with cerebrotendinous xanthomatosis: two retrospective cohort studies. Neurol Sci. (2019). doi: 10.1007/s10072-019-04169-8

5. Salen G, Steiner RED. Pidemiology diagnosis, and treatment of cerebrotendinous xanthomatosis (CTX). J Inherit Metab Dis. (2017) 40:771-81. doi: 10.1007/s10545-017-0093-8

6. Clayton PT, Verrips A, Sistermans E, Mann A, Mieli-Vergani G, Wevers R. Mutations in the sterol 27-hydroxylase gene (CYP27A) cause hepatitis of infancy as well as cerebrotendinous xanthomatosis. J Inherit Metab Dis. (2002) 25:501-13. doi: 10.1023/A:1021211520034

7. Gong JY, Setchell KDR, Zhao J, Zhang W, Wolfe B, Lu Y, et al. Severe neonatal cholestasis in cerebrotendinous xanthomatosis: genetics, immunostaining, mass spectrometry. J Pediatr Gastroenterol Nutr. (2017) 65:561-8. doi: 10.1097/MPG.0000000000001730

8. Berginer VM, Gross B, Morad K, Kfir N, Morkos S, Aaref S, et al. Chronic diarrhea and juvenile cataracts: think cerebrotendinous xanthomatosis and treat. Pediatrics. (2009) 123:143-7. doi: 10.1542/peds.2008-0192

9. Degos B, Nadjar Y, Amador Mdel M, Lamari F, Sedel F, Roze E, et al. Natural history of cerebrotendinous xanthomatosis: a paediatric disease diagnosed in adulthood. Orphanet J Rare Dis. (2016) 16:41. doi: 10.1186/s13023-0160419-x

10. Mignarri A, Gallus GN, Dotti MT, Federico A. A suspicion index for early diagnosis and treatment of cerebrotendinous xanthomatosis. J Inherit Metab Dis. (2014) 37:421-9. doi: 10.1007/s10545-013-9674-3

11. Yahalom G, Tsabari R, Molshatzki N, Ephraty L, Cohen H, Hassin-Baer S. Neurological outcome in cerebrotendinous xanthomatosis treated with chenodeoxycholic acid: early versus late diagnosis. Clin Neuropharmacol. (2013) 36:78-83. doi: 10.1097/WNF.0b013e318288076a diagnosis and treatment is important to prevent irreversible systemic and neurological damage.

\section{INFORMED CONSENT}

The patient's parents have provided written informed consent to genetic testing and publication of the case report.

\section{AUTHOR CONTRIBUTIONS}

ID and CA participated in the design of the work and wrote the draft of the article. AM and MTD contributed to the design of the work. GN designed the work and critically revised it. MDP performed plasma cholestarol analysis. GG and MN performed urinary bile acids profile. All authors have approved the final version of the article.

\section{FUNDING}

The authors received no specific funding for this work. This paper was partially supported by a contribution from the Italian Ministry of Health (Ricerca Corrente-2020 Grant).

12. Mandia D, Chaussenot A, Besson G, Lamari F, Castelnovo G, Curot J, et al. Cholic acid as a treatment for cerebrotendinous xanthomatosis in adults. J Neurol. (2019) 266:2043-50. doi: 10.1007/s00415-01909377-y

13. Yang $\mathrm{CH}$, Perumpail BJ, Yoo ER, Ahmed A, Kerner Jr J. Nutritional needs, and support for children with chronic liver disease. Nutrients. (2017) 9:1127 doi: 10.3390/nu9101127

14. Mignarri A, Magni A, Del Puppo, Gallus GN, Bjorkhem I, Federico A, et al. Evaluation of cholesterol metabolism in cerebrotendinous xanthomatosis. J Inherit Metab Dis. (2016) 39:75-83. doi: 10.1007/s10545-0159873-1

15. Kuriyama M, Fujiyama J, Kasama T, Osame M. High levels of plant sterols and cholesterol precursors in cerebrotendinous xanthomatosis. J Lipid Res. (1991) 32:223-9.

16. Pierre G, Setchell KDR, Blyth J, Preece MA, Chakrapani A, McKiernan P. Prospective treatment of cerebrotendinous xanthomatosis with cholic acid therapy. J Inherit metab Dis. (2008) 31 (Suppl. 2) (S241-5). doi: 10.1007/s10545-008-0815-Z

17. Setchell KDR, O’ Connell N, Russell DW. A unique case of cerebrotendinous xanthomatosis presenting in infancy with cholestatic liver disease further highlights bile acid synthetic defects as an important category of metabolic liver disease (abstract). In: Falk Symposium 120. XVI International Bile Acid Meeting (2000)

18. Huidekoper HH, Vaz FM, Verrips A, Bosch AM. Hepatotoxicity due to chenodeoxycholic acid supplementation in an infant with cerebrotendinous xanthomatosis: implications for treatment. Eur J Pediatr. (2016) 175:1436. doi: 10.1007/s00431-015-2584-7

19. Setchell KD, Dumaswala R, Colombo C, Ronchi M. Hepatic bile acid metabolism during early development revealed from the analysis of human fetal gallbladder bile. J Biol Chem. (1988) 263:16637-44.

20. Von Bahr S, Björkhem I, Van't Hooft F, Alvelius G, Nemeth A, Sjövall $\mathrm{J}$ et al. Mutation in the sterol 27-hydroxylase gene associated with fatal cholestasis in infancy. J Pediatr Gastroenterol Nutr. (2005) 40:4816. doi: 10.1097/01.MPG.0000150419.23031.2A

21. Heubi JE, Bove KE, Setchell KDR. Oral cholic acid is efficacious and well tolerated in patients with bile acid synthesis and zellweger spectrum disorders. J Pediatr Gastroenterol Nutr. (2017) 65:321-26. doi: 10.1097/MPG.0000000000001657 
22. Duell PB, Salen G, Eichler FS, DeBarber AE, Connor SL, Casaday $\mathrm{L}$, et al. Diagnosis, treatment, and clinical outcomes in 43 cases with cerebrotendinous xanthomatosis. J Clin Lipidol. (2018) 12:116978. doi: 10.1016/j.jacl.2018.06.008

23. Van Heijst AFJ, Verrips A, Wevers RA, Cruysberg JR, Renier WO, Tolboom J. Treatment and follow-up of children with cerebrotendinous xanthomatosis. Eur J Pediatr. (1998) 157:313-6. doi: 10.1007/s004310050818

24. James E Heubi JE, Setchell KDR. Open-label phase 3 continuation study of cholic acid in patients with inborn errors of bile acid synthesis. J Pediatr Gastroenterol Nutr. (2020) 70:423-9. doi: 10.1097/MPG.0000000000002618

25. Beppu T, Seyama Y, Kasama T, Serizawa S, Yamakawa T. Serum bile acid profiles in cerebrotendinous xanthomatosis. Clin Chim Acta. (1982) 118:16775. doi: 10.1016/0009-8981(82)90004-3

26. Salen G, Meriwether TW, Nicolau G. Chenodeoxycholic acid inhibits increased cholesterol and cholestanol synthesis in patients with cerebrotendinous xanthomatosis. Biochem Med. (1975) 14:57-74. doi: 10.1016/0006-2944(75)90020-4

27. Gonzales E, Matarazzo L, Franchi-Abella S, Dabadie A, Cohen J, Habes D, et al. Cholic acid for primary bile acid synthesis defects: a life-saving therapy allowing a favorable outcome in adulthood. Orphanet J Rare Dis. (2018) 13:190. doi: 10.1186/s13023-018-0920-5

28. Moghadasian MH, Salen G, Frohlich JJ, Scudamore CH. Cerebrotendinous xanthomatosis: a rare disease with diverse manifestations. Arch Neurol. (2002) 59:527-9. doi: 10.1001/archneur.59.4.527
29. Stelten BML, Huidekoper HH, van de Warrenburg BPC, Brilstra EH, Hollak CEM, Haak HR, et al. Long-term treatment effect in cerebrotendinous xanthomatosis depends on age at treatment start. Neurology. (2019) 92:e83e95. doi: 10.1212/WNL.0000000000006731

30. DeBarber AE, Kalfon L, Fedida A, Fleisher Sheffer V, Ben Haroush S, Chasnyk N, et al. Newborn screening for cerebrotendinous xanthomatosis is the solution for early identification and treatment. J Lipid Res. (2018) 59:2214-22. doi: 10.1194/jlr.M087999

31. Vaz FM, Bootsma AH, Kulik W, Verrips A, Wevers RA, Schielen PC, et al A newborn screening method for cerebrotendinous xanthomatosis using bile alcohol glucuronides and metabolite ratios. J Lipid Res. (2017) 58:10027. doi: 10.1194/jlr.P075051

Conflict of Interest: The authors declare that the research was conducted in the absence of any commercial or financial relationships that could be construed as a potential conflict of interest.

Copyright (c) 2020 Degrassi, Amoruso, Giordano, Del Puppo, Mignarri, Dotti, Naturale and Nebbia. This is an open-access article distributed under the terms of the Creative Commons Attribution License (CC BY). The use, distribution or reproduction in other forums is permitted, provided the original author(s) and the copyright owner(s) are credited and that the original publication in this journal is cited, in accordance with accepted academic practice. No use, distribution or reproduction is permitted which does not comply with these terms. 